\title{
Diagnostic and therapeutic strategiesfor resistant arterial hypertension - focus on countries with emerging economies
}

\author{
Zhanatbekova $\mathrm{AK}^{1,3}$, Karazhanova $\mathrm{LK}^{1}$, Begalina $\mathrm{AM}^{2}$, Filipova $\mathrm{S}^{3}$ \\ Department of Internal Medicine, Semey State Medical University, Semey, Kazakhstan. aselker@mail.ru
}

\begin{abstract}
Arterial hypertensionis an important worldwide health problem. Its relevance relatesboth to the high incidence and prevalence in all adult communities and to the high risk of serious and potentially fatal cardiovascular events dueto hypertension. Resistant hypertension is defined as a blood pressure (BP) remaining above goal (>140/90 mm Hg) despite the use of at least 3 optimally dosed antihypertensive drugs from different classes, with one of the drugs being a diuretic. The exact prevalence of $\mathrm{RH}$ is unknown, but it is generally estimated at $10-20 \%$ of hypertensive patients. The aim of this review article is to address several important issues: (1) How to diagnose true RH ? (2) What is the optimal state-of-art management of RH in the light of the most recent scientific evidence and what is the role of various medical specialties in this process ? (3) Are there any country specific issues related to diagnosing and treating of $\mathrm{RH}$ in Kazakhstan and if so, how to tackle them ? Long-lasting resistant hypertension increases by $50-80 \%$ the risk of major cardiovascular events (myocardial infarction, stroke) and end-organ damage. (heart failure, vascular dementia, chronic kidney disease). Adherence to well chosen therapy is the key factor in achieving blood pressure control and this must be based on adequate patient education and universal access to drug therapy. Thus, early recognition and appropriate management of $\mathrm{RH}$ must be among the top priorities of all public health initiatives to reduce the burden of cardiovascular diseases (Tab. 2, Fig. 1, Ref. 31). Text in PDF www.elis.sk.

Key words:resistant arterial hypertension, diagnosis, treatment.
\end{abstract}

\section{Introduction}

Albeithypertensionis in focus of cardiovascular research for several decades, resistant hypertension (RH) continues to be a serious public healthcare problem with adverse medical, social and economic impact. Worldwide, $14 \%$ of all deaths can be attributed to hypertension (1). Due to limitations in conducting epidemiologic studies on this subject, the exact prevalence of RH is not exactly known, but it is in general estimated that $10-18 \%$ of hypertensive patients have RH. The process of correct diagnosis of resistant hypertension relies on several key steps:

- Exclusion of pseudo-resistance focusing on 2 aspects:

- Evaluation of adherence to therapy and life-style recommendation (such as salt intake, alcohol consumption and others)

- Recognition of "white coat" hypertension

- Reliable exclusion of all forms of secondary hypertension.

In both developed and emerging econonomy countries, the control rate for hypertension is to low, especially in the light of

${ }^{1}$ Department of Internal Medicine, Semey State Medical University, Semey, Kazakhstan ${ }^{2}$ Department of PostgraduateEducation, Semey State Medical University, Semey, Kazakhstan ${ }^{3}$ Department of Cardiology and Angiology, School of Medicine, Slovak Medical University, Bratislava, Slovakia

Address for correspondence: A.K. Zhanatbekova, MD, Department of Internal Medicine, Semey State Medical University, 071400 Shakerimstreet 15-275, Semey City, Kazakhstan. availability of highly effective and safe drugs for hypertension. Poor adherence to medication is the primary reason. Unrecognized pseudo-resistant hypertension accounts probably for a significant proportion of patients wrongly diagnosed as „resistant“hypertension. Kazakhstan is in the midst of emerging from a Soviet Union state to a market economy. Remarkable progress has been achieved in the last years with establishing specialized cardiovascular centers in major cities across the country with a central highly specialized tertiary care center in the capital Astana. However, the burden of cardiovascular disease in Kazakhstan can be reduced only by efficacious diagnostic and therapeutic measures on a wide population basis. As physicians - cardiologists practicing in Kazakhstan, it is our aim to focus the problem of resistant hypertension in a wider context of arterial hypertension management in this country.

It was recently reported that according to estimates based on data from the European Society of Cardiology, the total number of patients with arterial hypertension in Kazakhstan may reach 6 million people - about $65 \%$ of the adult population of the country. Since the overall proportion of RH might be up to $18 \%$, there are possibly almost 1 million of patients with RH in Kazakhstan (2).

\section{Definition of resistantarterialhypertension}

According to the statement from the American Heart Association (AHA) defined resistant hypertension as a blood pressure (BP) remaining above goal $(>140 / 90 \mathrm{mmHg})$ despite the use of at least 3 optimally dosed antihypertensive drugs from different 
classes, with one of the drugs being a diuretic. (3) The National Institute for Health and Clinical Excellence guideline suggests that the 3 drugs should be an angiotensin-converting enzyme inhibitor or angiotensin blocker plus a calcium channel blocker plus a thiazide-type diuretic (4). According to 2013 ESH/ESC Guidelines for the management of arterial hypertension, hypertension is defined as resistant to treatment when a therapeutic strategy that includes appropriate lifestyle measures plus a diuretic and two other antihypertensive drugs belonging to different classes at adequate doses (but not necessarily including a mineralocorticoid receptor antagonist) fails to lower SBP and DBP values to target $<140$ and $90 \mathrm{mmHg}$, respectively (5). By extension of these definitions the spectrum of resistant hypertension alsoincludes all patients who need four or more drugs to control theirblood pressure.

\section{Epidemiology of resistant and pseudo-resistant arterial hy- pertension}

The exact prevalence of RH is unknown, in part probably due to its arbitrary definition. However, smaller studies have estimated prevalence from $5 \%$ in general medical practice up to $50 \%$ among patients in specialized nephrology clinics (6). An analysis of US National Health and Nutrition Examination Survey data suggests that among hypertensive adults treated with drugs, approximately $13 \%$ have RH. A recent study of RH in Spain found a similar rate of $12 \%$ (7). Data from the BP-CARE study provided evidence that the prevalence of true RH in Central and Eastern European countries is similar to that found in Western Europe and USA (8). The 2013 ESH/ESC Guidelines for the management of arterial hypertension report the prevalence of RH in the range from 5 to $30 \%$ of the overall hypertensive population, with figures around $10 \%$ probably representing the true prevalence.

Corresponding systematic data concerning Kazakhstan, countries of the Commonwealth of Independent States (former USSR) and countries in Central Asia are not available. The prevalence of RH in Japan reported in J-HOME study was $13 \%$ (8). Surprisingly, the HOT-CHINA Study revealed only $2 \%$ resistant hypertension in Chinese hypertensive (7), mainly occurring in overweight male patients with metabolic syndrome. Nevertheless, these data are in sharp contrast with several other reports on limited hypertension control in China. Li et al (11) in the report based on screening of 16 364 adult rural residents identified $44 \%$ of the population having hypertension but only $3.9 \%$ of hypertensive patients to achieve target BP value (!). The authors conclude that prevalence of hypertension in rural China is high, but levels of awareness, treatment and control are unacceptably low.Furthermore, Economicaspects of accesibility of drugtherapyimpactsignificantlytheadherence to therapy, and thisistrueforbothdeveloped and developingcountries(12). A USbased report on over 300000 patientsdemonstratedthatzeroout-ofpocketcopaymentsfordrugsimprovesadehrence by $40 \%$ (in comparison to copayments in theranging of USD 1-9). So availability of effectivetherapieswithminimalcostsforthepatientplay a crucial role in eliminating ,resistant" hypertension by increasingadherence to hypertensiontherapyand reducingcostsforteratingseriouscomplication of hyperetensivedisease (13). In tran- sient economies like Kazakhstan, economic aspects of adherence to drug therapy might play an even more important role. Out-ofpocket spending on drug therapy for cardiovascular disease is up to $38 \%$ higher than for other diseasesin this country (14). Such spending might negatively impact patient's family budget especially in the rural areas. Asa result, patient's adherence to therapy might be compromised with the resulting insufficient blood pressure control. These issues deserve special attention in designing public cardiovascular healthcare policies.

\section{Etiology of RH, pseudo-resistance and factors of resistance to treatment}

The etiology of RH is almost always multifactorial. Before a patient is considered to be diagnosedwith $\mathrm{RH}$, pseudo-resistant hypertension must be excluded(Tab.1). This condition, which is frequently confused with RH, is characterized by BP levels persistently above normal values at clinic measurements in patients who otherwise do nothave resistant hypertension (15).However,

Tab. 1. Possible causes of pseudo-resistant hypertension (adapted from 16).

\begin{tabular}{l}
\hline 1) Errors at BP measurements: \\
\hline - use of small cuffs on large arms, with inadequate compression of \\
the vessel \\
• pseudohypertension, i.e. marked arterial stiffening (more common \\
in the elderly, especially with heavily calcified arteries), which pre- \\
vents occlusion of the brachial artery. \\
\hline 2) Low patient's adherence due to: \\
- the lack of awareness about the problem of hypertension \\
- low cultural level of the patient \\
- large number of prescribed drugs or tablets \\
- the presence of side effects \\
- economic factors \\
- mental disorders
\end{tabular}

3) Irrational prescribing regime:

- inadequate frequency of administration

- irrational combinations

- inadequate doses

4) The absence or lack of lifestyle modification:

- obesity

- alcohol abuse

- smoking

- excessive use of salt

5) Volume overload caused by:

- renal failure

- the use of antihypertensive drugs

- excessive use of salt and fluid

- inadequate diuretic therapy

\begin{tabular}{l} 
6) Drug induced: \\
\hline - administration of drugs or agents that increase blood pressure (cor- \\
ticosteroids, anabolic steroids, non-steroidal anti-inflammatory drugs \\
(NSAIDs), sympathomimetics, oral contraceptives, erythropoietin, \\
anoretics, chlorpromazine, monoamine oxidase inhibitors (MAOIs), \\
tricyclic antidepressants \\
\hline 7) Special conditions: \\
\hline - insulin resistance \\
- sleep apnea \\
- white coat hypertension \\
\hline
\end{tabular}


the most frequent reason for pseudo-resistance is poor adherence to drug therapy and/or suboptimal choice of drugs. Table 1 Summarizes the various reasons for psedo-resistance of hypertension to therapy. It should pointed out that a frequently neglected reason for pseudo-resistance and renal damage is concomitant use (or even abuse) of Non-steroidal anti-inflammatory drugs (NSAIDs), including selective cyclooxygenase (COX) 2 inhibitors. Most NSAIDs increase the BP through volume and sodium retention, mediated by inhibition of vasodilating prostaglandins in the kidney.

Important insight on the problem of adherence to cardiovascular therapy was recently presented in the meta-analysis of prospective epidemiological studies (17). According to these results prevalence of good adherence for antihypertensive agents was $59 \%$. Poor patient compliance to therapy, inadequate doses of antihypertensive drugs, inadequate choice of combinations of antihypertensive drugs, poor office blood pressure measurement technique, and having to pay for costs of drugs are all important factors associated with pseudoresistanthypertension.

In evaluating resistant hypertension, white coat hypertension (BP $>140 \mathrm{~mm} \mathrm{Hg}$ but a normal home BP or 24 hour ambulatory blood pressure monitoring - ABPM) must also be excluded. White-coat effect is the difference between office BP and ABPM or home BP measurements and can be calculated as the mean office BP minus mean daytime ambulatory BP.

In conclusion, following factors importantly contribute to RH: obesity, excess dietary sodium, excess alcohol intake, cocaine abuse, amphetamines, non-steroidal anti-inflammatory drugs, contraceptive hormones, adrenal steroid hormones, sympathomimetic drugs (nasal decongestants and diet pills), erythropoetin, licorice, herbal supplements (ephedra), progressive renal insufficiency, and inadequate diuretic therapy.

\section{Cardiovascular risk and target organ damage in resistant hy- pertension}

Cardiovascular (CV) complications such as myocardial infarction, heart failure, stroke and renal failure are related to both the degree and the duration of $\mathrm{BP}$ increase. $\mathrm{RH}$ is associated with a higher risk of $\mathrm{CV}$ complications and a higher prevalence of target organ damage (TOD). The relationship between CV disease and TODis bidirectional. Persistently elevated BP in RH may cause CV structural and functional alterations such as: development of left ventricular hypertrophy, arterial stiffness, atherosclerotic plaques, microvascular disease and renal dysfunction. All these, in turn, may render hypertension more difficult to control (18).Recently, Daugherty et al. (19) confirmed the high rate of CV events (CV death, myocardial infarction, heart failure, stroke or chronic kidney disease) in patients with RH. Among 205750 patients with incident hypertension, $1.9 \%$ developed $\mathrm{RH}$ within a median of 1.5 years from the initial treatment. These resistant hypertensive patients were older, more often men and more frequently diabetics than were the nonresistant patients. CV events rates were significantly higher in those with $\mathrm{RH}$ as compared with those without (18.0\% vs. $13.5 \%, \mathrm{P}<0.001$ ), and the hazard ratio was 1.47 (confidence interval (CI), 1.33-1.62) after adjusting for patient and clinical characteristics. The presence of TODin different vascular districts, including the heart and kidney, may explain the increased resistance to medical treatment. Furthermore, the presence of TOD is associated with a very high absolute CV risk, as recently underlined; thus, an urgent need exists to appropriately recognize and identify patients with RH and to achieve as soon as possible both adequate BP control and TOD regression(19).

\section{Exclusion of secondary forms of hypertension}

The major part of diagnostic process of $\mathrm{RH}$ is focused on the exclusion of secondary forms of hypertension. Studies indicate that $5-10 \%$ of RH patients have an underlying secondary cause for their elevated $\mathrm{BP}(20)$. Secondary hypertension is a type of hypertension with aknown and potentially correctable underlying cause. A secondary etiology may be suggested by various nonspecific and specific symptoms (e.g., flushing and sweating suggestive of pheochromocytoma), physical examination findings (e.g., a renal bruit suggestive of renal artery stenosis, further neede to be confirmed by abdominal ultrasound), or laboratory abnormalities (e.g., hypokalemia suggestive of aldosteronism) (21). The most frequent causes of secondary RH include primary hyperaldosteronism, obstructive sleep apnea, renal artery stenosis, renal parenchymal disease, aortic coarctation, Cushing's syndrome, pheochromocytoma, hyperthyroidism, hypothyroidism, and intracranial tumors. Summary of possible etiologies of secondary hypertension is in table 2 .

Primary hyperaldosteronism (PHA) is the most common cause of secondary hypertension and is a frequent contributor to treatment resistance. The prevalence of PHA is greater than previously thought, partially because hypokalemia and adrenal tumors are no longer necessary criteria for the diagnosis of PHA. The prevalence of PHA is even higher in patients with resistant high BP, mostly $17 \%$ to $22 \%$ in multiple studies. Aldosterone-renin ratio is considered the most reliable test for screening of PHA, but false-positive and false-negative results may occur depending on posture, time of the day, salt intake, plasma potassium, and concurrent medications. Medication such as diuretics, angiotensin-converting enzyme (ACE) inhibitors and angiotensin receptor blockers (ARBs) can mask the diagnosis of PA by causing false negatively low aldosterone-renin ratios. However, although interfering medications should be ideally stopped before screening for PHA, the risk of stopping medications in patients with RH needs to be carefully assessed in order to avoid loss of hypertension control. If aldosteronerenin ratio is positive, PHA has to be confirmed by fludrocortisone suppression test, oral sodium loading, and saline infusion testing or captopril challenge test. After confirmation of PHA, lateralization of the source of the excessive aldosterone secretion demonstrated by adrenal vein sampling is critical to guide the management of PHA. Unilateral adrenalectomy usually has a major beneficial effect in patients with confirmed PHA and lateralization of aldosterone overproduction to one adrenal on adrenal venous sampling, not only on hypertension control, but also on quality of life, reduction in risk of cardiovascular events and renal organ damage. 
Tab. 2. Signs and symptoms that suggest specific causes of secondary hypertension (Adapted from 21).

\begin{tabular}{|c|c|c|}
\hline Signs/symptoms & $\begin{array}{l}\text { Possible secondary hypertension } \\
\text { cause }\end{array}$ & Diagnostic test options \\
\hline Hypokalemia & hyperaldosteronism & $\begin{array}{l}\text { Renin and aldosterone levels to calculate aldosterone/ } \\
\text { /renin ratio }\end{array}$ \\
\hline $\begin{array}{l}\text { Apneic events during sleep } \\
\text { Daytime sleepiness } \\
\text { Snoring }\end{array}$ & Obstructive sleep apnea & $\begin{array}{l}\text { Polysomnography (sleep study) } \\
\text { Sleep Apnea Clinical Score with nighttime pulse oxi- } \\
\text { metry }\end{array}$ \\
\hline $\begin{array}{l}\text { Increase in serum creatinine concentration ( } \geq 0.5 \text { to } 1 \mathrm{mg} \\
\text { per } \mathrm{dL}[44.20 \text { to } 88.40 \mu \mathrm{mol} \text { per } \mathrm{L}] \text { ) after starting } \\
\text { angiotensin-converting enzyme inhibitor or angio- } \\
\text { tensin receptor blocker } \\
\text { Renal bruit }\end{array}$ & Renal artery stenosis & $\begin{array}{l}\text { Computed tomography angiography } \\
\text { Doppler ultrasonography of renal arteries } \\
\text { Magnetic resonance imaging with gadolinium con- } \\
\text { trast media }\end{array}$ \\
\hline $\begin{array}{l}\text { Arm to leg systolic blood pressure difference }>20 \mathrm{mmHg} \\
\text { Delayed or absent femoral pulses } \\
\text { Murmur }\end{array}$ & Coarctation of the aorta & $\begin{array}{l}\text { Magnetic resonance imaging (adults) } \\
\text { Transthoracic echocardiography (children) }\end{array}$ \\
\hline $\begin{array}{l}\text { Bradycardia/tachycardia } \\
\text { Cold/heat intolerance } \\
\text { Constipation/diarrhea } \\
\text { Irregular, heavy, or absent menstrual cycle }\end{array}$ & Thyroid disorders & Thyroid-stimulating hormone \\
\hline $\begin{array}{l}\text { Flushing } \\
\text { Headaches } \\
\text { Labile blood pressures } \\
\text { Orthostatic hypotension } \\
\text { Palpitations } \\
\text { Sweating } \\
\text { Syncope }\end{array}$ & Pheochromocytoma & $\begin{array}{l}\text { 24-hour urinary fractionated metanephrines } \\
\text { Plasma free metanephrines }\end{array}$ \\
\hline $\begin{array}{l}\text { Buffalo hump } \\
\text { Central obesity } \\
\text { Moon facies } \\
\text { Striae }\end{array}$ & Cushing syndrome & $\begin{array}{l}\text { 24-hour urinary cortisol } \\
\text { Late-night salivary cortisol } \\
\text { Low-dose dexamethasone suppression }\end{array}$ \\
\hline
\end{tabular}

\section{Obstructive Sleep Apnea Syndrome (OSAS)}

OSAS is characterized beside snoring by sleepiness during daytime, frequent nocturnal micro-arousals, morningasthenia and frequent morning headaches. More specifically, OSAS is a sleep disorder that is characterized by at least10 apnea-hypopnea events during each hour of sleep.It is common among patients with RH, but OSAScan'tbe counted as a typical form of "secondary hypertension (22). The gold standard for diagnosis is polysomnography (sleep study), but clinical assessment tools such as the Epworth Sleepiness or the Sleep Apnea Clinical Score coupled with nighttime pulse oximetry may be sufficient for the diagnosis of moderate to severe OSA, particularly if cost and availability are limiting factors. In some patients, however, RH may be the only sign for OSA. In one study of patients with $\mathrm{RH}, 83 \%$ were diagnosed with unsuspected OSA on the basis of polysomnogram results (23). Therefore, a polysomnogram should be considered in patients with RH. In those found to have OSA, treatment with continuous positive airway pressure may help improve BP control.

\section{Renal Artery Stenosis}

In younger adults, particularly women, renal artery stenosis is one of the most common causes of secondary hypertension. The finding of an audible, high-pitched, holosystolic renal artery bruit would raise suspicion and warrant diagnostic imaging. Renal Dopplerultrasound examination can be used, which provides usefulin- formation regarding blood flow. In positive case renal angiography, computed tomography $(\mathrm{CT})$ angiography or magnetic resonance imaging (MRI) used to visualize and quantify stenosis. Although identifying renal artery stenosis caused by fibromuscular dysplasia is important, identifying renal artery stenosis caused by atherosclerosis (usually in older adults) is less critical because evidence does not show a benefit of percutaneous revascularization over medical management (ie. blood pressure control, statin, antiplatelet agents) (7).

Based on the above observations, a simplified algorithmfor diagnosis of resistant arterial hypertension can be proposed (Fig. 1).

After correct diagnosis of RH the physician should modify existing therapy or start therapy. The ultimate goal of treating high $\mathrm{BP}$ is prevention of hypertensive end-organ damage and reduction of cardiovascular morbidity and mortality.

\section{Combination therapy}

Clinical studies have revealed that an effective way to control RH can be based on an early initiation of antihypertensive treatment with drug combinations, as suggested by current guidelines. Combination strategy should be based on the use of drugs with synergistic mechanisms. Available evidence demonstrated a better BP control by combining drugs able to modulate the abnormal activation of the renin-angiotensin system, including ACE inhibitors or ARBs, with vasodilating agents, such as $\mathrm{CCBs}$, alfa-blockers or diuretic (thiazide) drugs (15). If tolerated, a regimen consisting of a thiazide diuretic, ACE inhibitor or ARB, and a long-acting calcium 


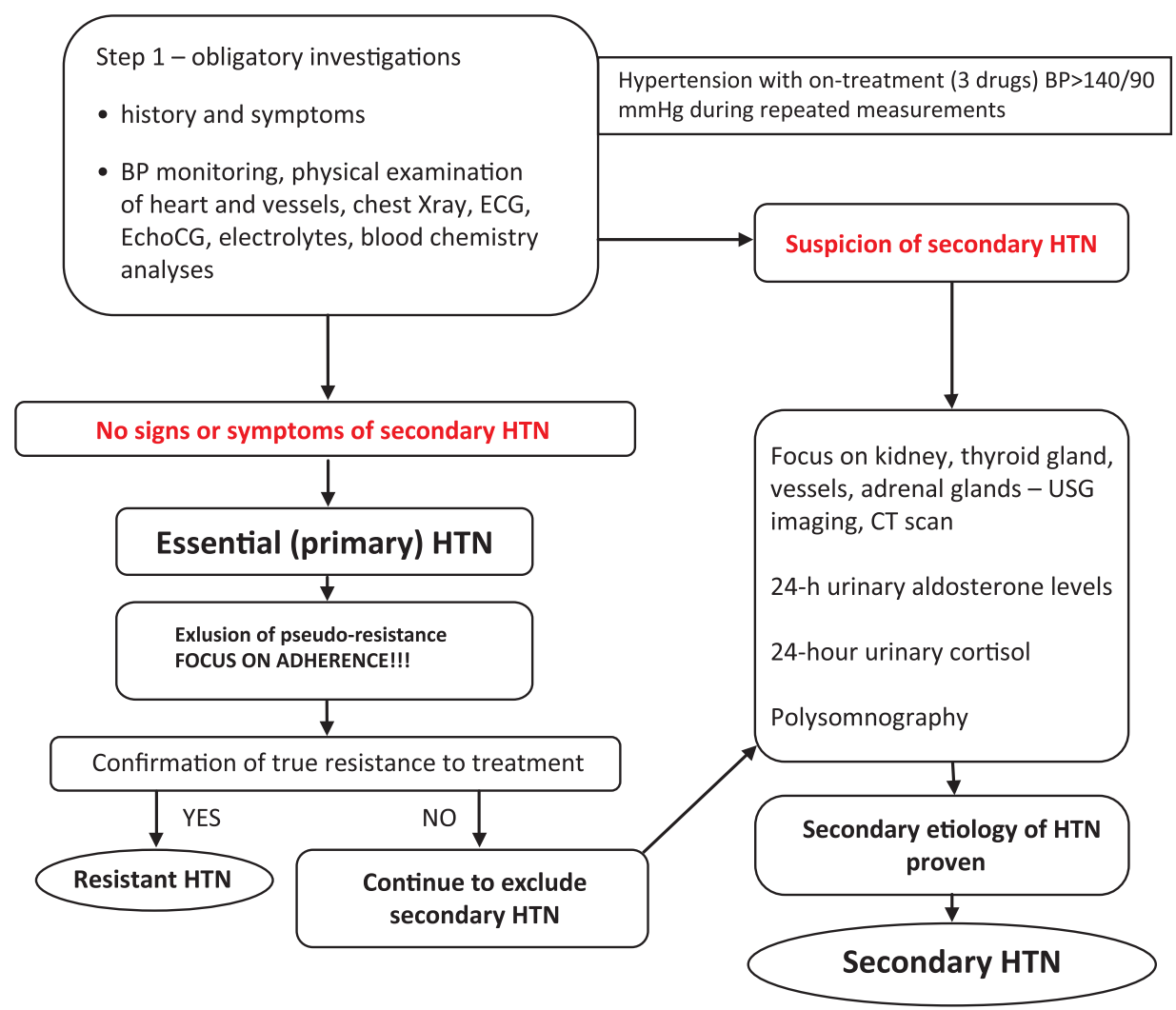

Fig. 1. Simplified diagnostic flow-chart algorithm for resistant arterial hypertension (=HTN)

channel blocker is recommended as the initial triple combination. This combination is generally effective and well tolerated and can be prescribed with the use of generic medications or with one or two pills with the use of combination products.

Given its superior efficacy, chlorthalidone is recommended for preferential use in patients who remained uncontrolled on less-potent thiazide diuretics. The renin-angiotensin-aldosterone system (RAAS) is the main regulator of sodium and fluid balance, and one of the major mechanisms involved in the pathogenesis of hypertension. Dual blockade of the RAAS with mineralocorticoid receptor antagonists, specifically spironolactone, in combination with an ACE inhibitor or ARB, is now routinely commonly used to treat RH $(24,25,26)$.

Management of patient with true RH should be started from lifestyle changes and combined drug therapy targeting the key factors in the pathogenesis of hypertension. Of course, the choice of drugs depends on concomitant diseases and condition of target organs. Best combination for regimen consisting of a thiazide diuretic, ACE inhibitor or ARB, and a long-acting calcium channel blocker is recommended as the initial triple combination. Spironolactone, in combination with an ACE inhibitor or ARB, is now increasingly used to effectively treat RH. (24)

\section{Interventional therapy of arterial RH}

In addition to the pharmacological treatment, two new therapy options are available. It is now known that autonomic control of the kidney is predominantly sympathetic, and mediated by a dense network of post-ganglionic sympathetic neurons in the kidney. Hypertension is characterized by excessive stimulation of this sympathetic neural network, evidenced by high rates of renal norepinephrine spillover into the circulation, and increased systemic sympathetic nerve firing, possibly modulated by afferent renal sensory nerves. This excessive sympathetic outflow to the kidney increases both renin release and tubular sodium reabsorption, and often reduces renal blood flow. In addition, afferent signals from the kidney directly contribute to neurogenic hypertension by modulating central sympathetic outflow. Afferent renal sympathetic nerves originate mostly from the renal pelvic wall and respond to either mechanoreceptors that detect stretch, or chemoreceptors that detect renal ischemia. These fibers, which have cell bodies in the ipsilateral dorsal root ganglia (T6-L4), ascend to the central nervous system, mainly to the hypothalamus, where they evoke functional changes and a central sympathetic response(27, 28). Surgical renal denervation, although highly effective in reducing blood pressure, was associated with a significant amount of side-effects and was rapidly replaced by better-tolerated medical therapy. Current pharmacologic strategies attempting to control blood pressure in patients with resistant hypertension are not always effective.

Recently, a catheter-based method to induce renal sympathetic denervation has been introduced into daily practice (27, $28,29)$. As a result, a succession of therapeutic approaches has 
targeted the sympathetic nervous system to modulate hypertension, with varying success. The rapid progress in catheter-based technologies occurring within the last 20 years facilitated the development of percutaneous catheter-based renal artery ablation that has emerged as a new approach to achieving blood pressure reduction in patients with resistant hypertension. Early results from the use of catheter-based renal denervation suggest significant reduction in blood pressure while maintaining the safety and efficacy of the method.

Renal denervation technique was initially tested in an openlabel pilot clinical study, SymplicityHTN-1, conducted with 45 patients with RH, with preserved renal function. Patients administrated, in average, 4.7 antihypertensive drugs and had mean baseline BP of 177/101 mmHg. Primary outcomes of the study were procedure safety and decrease of casual BP. Positive results of this initial pilot study stimulated further studies. In the SymplicityHTN-2, randomized, prospective, multicenter study, 106 patients with resistant hypertension were randomized for renal denervation ( $\mathrm{n}=52$, initial mean $\mathrm{BP}$ of $178 / 96 \mathrm{mmHg}$ ) or maintenance of the previous drug therapy $(n=54$, initial mean BP of $178 / 97 \mathrm{mmHg}$ ), having as main outcome the modification of casual BP in six months and as secondary outcomes the procedure safety, occurrence of cardiovascular outcomes, and additional measures of BP after six months (29). These results stimulated the clinical use of renal sympathetic denervation with over 10000 patients treated across the world. Unfortunately, the recently presented SymplicityHTN-3 study did not confirm the initial enthusiasm (30). This blinded trial did not show a significant reduction of systolic blood pressure in 535 randomized patients with resistant hypertension six months after renal-artery denervation as compared with a sham control. So for the time being, available clinical data on percutaneous catheter-based renal artery sympathetic denervation for treatment of resistant hypertension are conflicting and further research is highly needed before a widespread use of this therapy can be recommended.

Another possible interventional approachto improve therapy in RH is carotid baroreceptor stimulation (31). Baroreceptors are stretch-sensitive fibers located primarily in the aortic arch and each of the carotid sinuses near the area where the common carotid artery bifurcates Experimental studies have clearly demonstrated that prolonged baroreflexactivation leads to sustained reductions in arterial pressure and heart rate. Chronic electrical stimulation of carotid sinus nerves via implanted devices in humans has recently been reported to reduce SBP and DBP in resistant hypertensive individuals. However, long-term observations have been performed only on limited number of patients and further data in patients with true $\mathrm{RH}$ are necessary to confirm the therapeutic potential of the procedure (31).

\section{Conclusion}

Unsatisfactory control of BP to target values in hypertensive patients continues to be a major clinical challenge in cardiovascular medicine. The proportion of patients with hypertension controlled to target values is highly variable across the world, reaching approximately only 4-30\%, with the lowest values achieved mainly in populations with low socio-economic status, frequently in rural areas. Identifying patients with pseudoresistant or truly resistant hypertension must be the key integral part of any program for improving cardiovascular health. Longlasting resistant hypertension increases by $50-80 \%$ the risk of major cardiovascular events and end-organ damage. Adherence to well chosen therapy is the key factor in achieving blood pressure control and this must be based on adequate patient education and universal access to drug therapy. Vast majority of patients with resistant hypertension can be successfully managed according to international guidelines with individualized combination drug therapy and lifestyle modification. Drugs with highest available scientific evidence must be administered for these patients and the combination must always comprise long-acting diuretics. The drug of choice areACE inhibitors / angiotensin receptor blockers, diuretics, calcium channel blockers, aldosterone antagonists and beta-blockers. Non-pharmacological interventional therapies currently play only a minor role in the management of resistant hypertension. Thus, early recognition, appropriate management and universal access to effective drugs with strong scientific evidence of efficacy and safety must be among the top priorities of all public health initiatives to reduce the burden of cardiovascular diseases.

\section{Acknowledgement}

This work was performed within the framework of the mutual collaboration agreement between the Semey State Medical University, Semey, Kazakhstan, and the Slovak Medical University, Bratislava, Slovakia. We would like to express our gratitude to the Department of Cardiology and Angiology of the School of Medicine of the Slovak Medical University and the European Excellent Center of Hypertension, National Cardiovascular Institute in Bratislava, Slovakia for the support in preparing the manuscript during the $\mathrm{PhD}$ curriculumod Dr. A. Zhanatbekova. Our thanks goes in particular to Assoc. Prof. Slavomira Filipova, $\mathrm{PhD}$ for her systematic mentorship and to Prof. Dr. Robert Hatala, PhD for revising the manuscript.

\section{References}

1. Lawes CM, Vander Hoorn S, Rodgers A.; International Society of Hypertension. Global burden of blood-pressure-related disease, 2001. Lancet 2008;371:1513-1518.

\section{2. http://lite.bnews.kz/ru/news/post/6006/}

3. Calhoun DA, Jones D, Textor S, Goff DC, Murphy TP, Toto RD, et al.Resistant hypertension: diagnosis, evaluation, and treatment: ascientific statement from the American Heart AssociationProfessional Education Committee of the Council for High BloodPressure Research. Circulation 2008; 24; 117: 510-26.

4. NICE clinical guideline 127. Hypertension: clinical management of primary hypertension in adults. Downloaded on Oct 26, 2013 at www. nice.org.uk/guidance/CG127 
5. ManciaG1, Fagard R, Narkiewicz KJ et al. 2013 ESH/ESC Guidelines for the management of arterial hypertension: the Task Force for the management of arterial hypertension of the European Society of Hypertension (ESH) and of the European Society of Cardiology (ESC). J Hypertens. 2013 31(7):1281-357

6. MakrisA1, Seferou M, Papadopoulos DP. Resistant hypertension workup and approach to treatment. Int J Hypertens. 2011, doi: $10.4061 / 2011 / 598694$.

7. VieraAJ, Neutze DM. Diagnosis of secondary hypertension: an agebased approach. Am Fam Physician. 2010 Dec 15;82(12):1471-8

8. BrambillaG1, Bombelli M, Seravalle G, et al. Prevalence and clinical characteristics of patients with true resistant hypertension in Central and Eastern Europe: data from the BP-CARE study.J Hypertens. 2013; 10):2018-24.

9. Nishikawa T1, Omura M, Saito J, Matsuzawa Y. The possibility of resistant hypertension during the treatment of hypertensive patients.Hypertens Res. 2013; 36:924-9.

10. Ma W1, Zhang Y; HOT-CHINA working group. Low rate of resistant hypertension in Chinese patients with hypertension: an analysis of the HOT-CHINA study. J Hypertens. 2013; 31:2386-90

11. Li H, Meng Q, Sun X, Salter A, et al. Prevalence, awareness, treatment, and control of hypertension in rural China: results from Shandong province. J Hypertens. 2010;28(3):432-438.

12. Yu B, Zhang $X$, Wang G. Full coverage for hypertensiondrugs in rural communities in China. Am J Manag Care. 2013;19:e22-9.

13. Briesacher BA, Andrade SE, Fouayzi H, Chan KA. Medication adherence and use of generic drug therapies. Am J Manag Care. 2009; 15:450456

14. Sari N1, LangenbrunnerJC: Consumer out-of-pocket spending for pharmaceuticals in Kazakhstan: implications for sectoral reform Health Policy Plan. 2001;16:428-34.

15. Volpe M1, Tocci G. Challenging hypertension: how to diagnose and treat resistant hypertension in daily clinical practice. Expert Rev CardiovascTher. 2010; 8:811-20.

16. Bezrodna L. Resistant (refractory) hypertension. Kiev, AMN, pp. 262-269.

17. Chowdhury R1, Khan H, Heydon E et al. Adherence to cardiovascular therapy: a meta-analysis of prevalence and clinical consequences. Eur Heart J 2013; 34: 2940-2948.

18. MuiesanML1, Salvetti M, Rizzoni D, et al. Resistant hypertension and target organ damage.Hypertens Res. 2013; 36:485-91
19. Daugherty SL1, Powers JD, Magid DJ et al. Incidence and prognosis of resistant hypertension in hypertensive patients. Circulation. 2012;125:1635-42.

20. Myat A1, Redwood SR, Qureshi AC, et al. Resistant hypertension. BMJ. 2012; 345:e7473. doi: 10.1136/bmj.e7473.

21. VieraAJ, Neutze DM. Diagnosis of secondary hypertension: an agebased approach.AmFam Physician. 2010 Dec 15;82(12):1471-8.

22. Somers VK, White DP, Amin R, et al. Sleep apnea and cardiovascular disease: an American HeartAssociation/American College of Cardiology Foundation Scientific Statement from the American Heart Association Council for High Blood Pressure Research Professional Education Committee, Council on Clinical Cardiology, Stroke Council, and Council on Cardiovascular Nursing. J Am CollCardiol 2008; 52: 686-717.

23. Logan AG, Perlikowski SM, Mente A, et al. High prevalence of unrecognized sleep apnoea in drug-resistant hypertension. J Hypertens 2001;19:2271-7.

24. PimentaE, Calhoun DA. Treatment of resistant hypertension. J Hypertens 2010, 28:2194-2195.

25. Dlesk A, Kamensky G, Stefanik M et al. Treatment efficiency of resistant hypertension in cardiologist's office. Bratisl Lek Listy 2014; 115 (1): 25-29.

26. Paiva L, Cachulo MC, Providencia R et al. Overview of resistant hypertension: A glimpse of the cardiologist's current standpoint. World J Cardiol2012; 4(9): 275-283.

27. GewirtzJR1, Bisognano JD. Catheter-based renal sympathetic denervation: a targeted approach to resistant hypertension. Cardiol J. 2011;18(1):97-102.

28. Polimeni A1, Curcio A, Indolfi C. et al.. Renal sympathetic denervation for treating resistant hypertension. Circ J. 2013; 77:857-63

29. Esler MD, Krum H, Sobotka PAet al. Renal sympathetic denervation in patients with treatment-resistant hypertension (the SYMPLICITYHTN-2 Trial): a randomised controlled trial. Lancet 2010;376:1903-1909

30. Bhatt DL, Kandzari DE, O'Neill WWet al. A controlled trial of renal denervation for resistant hypertension. N Engl J Med. 2014;370 (15):1393401 Epub 2014 Mar 29

31. LohmeierTE1, Iliescu R. Chronic lowering of blood pressure by carotid baroreflex activation: mechanisms and potential for hypertension therapy. Hypertension.2011 ; 57(5):880-6.

Received December 6, 2013. Accepted February 2014. 